\title{
Erarbeitung eines neuen Verfahrenskonzeptes zur Erzeugung innovativer Mineralprodukte aus sekundären Rohstoffquellen
}

\author{
Valentin Spieß ${ }^{1}$, Johannes Theiss ${ }^{2}$ und Helmut Flachberger ${ }^{1}$ \\ ${ }^{1}$ Lehrstuhl für Aufbereitung und Veredlung, Department Mineral Resources Engineering, Montanuniversität \\ Leoben, Leoben, Österreich

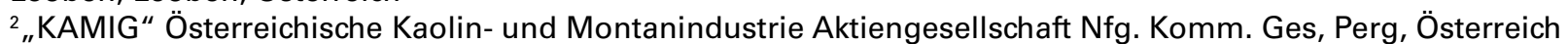

Eingegangen 15. Oktober 2020; angenommen 19. Oktober 2020; online publiziert 11. November 2020

\begin{abstract}
Zusammenfassung: Die in der Vergangenheit angefallenen und in der Zukunft anfallenden Aufbereitungsabgänge der KAMIG Österreichische Kaolin- und Montanindustrie Aktiengesellschaft Nfg. Komm. Ges. sollen nutzbar gemacht werden. Dazu wurde das strategische Forschungsprojekt „Erarbeitung eines neuen Verfahrenskonzeptes zur Erzeugung innovativer Mineralprodukte aus sekundären Rohstoffquellen" mit dem Lehrstuhl für Aufbereitung und Veredlung der Montanuniversität Leoben initiiert. Die Entwicklung eines Verfahrens zur vollständigen Nutzung der Minerale aus den unterschiedlichen Halden und Schlickerteichen der KAMIG ist das Ziel. Konkret sollen neben marktfähigen Kaolinprodukten auch hochqualitative Quarzprodukte für beispielsweise die Glasindustrie, Feldspatprodukte sowie Seltene-Erden-(Vor-)Konzentrate hergestellt werden können.
\end{abstract}

Schlagworte: Aufbereitung, Kaolin, Quarz, Feldspat, Sekundärrohstoff, Glassand, Seltene Erden

Process Development for Innovative Mineral Products (Kaolin, High-grade Silica and Feldspar) from Anthropogenic Deposits

Abstract: During mining activities at the sites of KAMIG (Österreichische Kaolin- und Montanindustrie Aktiengesellschaft Nfg. Komm. Ges.) substantial amounts of tailings have been produced over the last century. To make use of the valuable mineral-contents, KAMIG KG

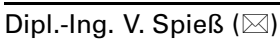

Lehrstuhl für Aufbereitung und Veredlung, Department Mineral

Resources Engineering,

Montanuniversität Leoben,

Franz-Josef-Str. 18

8700 Leoben, Österreich

valentin.spiess@unileoben.ac.at and the Chair of Mineral Processing initiated the strategic research project "Process development for innovative mineral products (Kaolin, high-grade Silica sand, Feldspar) from anthropogenic deposits". The aim of this project is to develop a process to beneficiate the Quartz-, Potassium-Feldspar, Kaolinite- as well as rare-earth-containing mineral phases from the anthropogenic deposits to marketable, high-quality concentrates in order to expand the product portfolio of KAMIG KG.

Keywords: Mineral processing, Kaolin, Quartz, Silica, Feldspar, Secondary raw materials, Glass sand, REE, Rare earth elements

\section{Projekthintergrund}

Kaolin, ein weißes, feinkörniges Gestein, welches sich aus feinstdispersen Kaolinitgruppenmineralen nebst den Bestandteilen des Vorläufergesteins (Kalifeldspat, Quarz, Glimmer, etc.) zusammensetzt, ist mit einer Welt-Jahresproduktion von 40,6 Mio. t mengenmäßig das bedeutendste Tonmineral (gefolgt von Bentonit mit 18,7 Mio. t/a), sowie eines der Industrieminerale mit der größten globaIen Nachfrage [1]. In Österreich wird seit etwa 200 Jahren an mehreren Standorten im Bezirk Perg/OÖ Rohkaolin abgebaut und aufbereitet, mit Spitzenwerten von etwa $180.000 \mathrm{t} / \mathrm{a}$ Rohkaolinförderung und -verarbeitung in den 1960er-Jahren [2].

Das bei der "KAMIG“ Österreichische Kaolin- und Montanindustrie Aktiengesellschaft Nfg. Komm. Ges. (nachfolgend mit KAMIG abgekürzt) zum Einsatz gelangende Aufbereitungsverfahren hat immer wieder Veränderungen erfahren, gegenwärtig besteht es aus den Teilprozessen "Waschen“ (mittels Schlämmtrommel und nachgeschalteten Schwerterwäschern), "Klassieren“ (Hydrozyklon-Gegenstromkaskaden und Überkorn-Kontrollsieben), „Ent- 
wässern“ und „Trocknen“. Zum Zeitpunkt der Inbetriebnahme der derzeit betriebenen Aufbereitungsanlage im Jahr 1982 war das Ziel die Erzeugung markttauglicher Kaolinprodukte, lediglich untergeordnet wurden die im ebenfalls im Rohkaolin enthaltenen Begleitminerale wirtschaftlich genutzt. Daher haben sich im Lauf der Zeit Aufbereitungsabgänge in der Größenordnung von mehreren Millionen Tonnen an den Standorten der KAMIG angesammelt.

Um das vorhandene Rohstoffpotential dieser anthropogenen Lagerstätten umfassender zu nutzen, wurde das Projekt „Erarbeitung eines neuen Verfahrenskonzeptes zur Erzeugung innovativer Mineralprodukte aus sekundären Rohstoffquellen" in Kooperation mit dem Lehrstuhl für Aufbereitung und Veredlung der Montanuniversität Leoben als strategischem Forschungspartner initiiert. Dieses hat zum Ziel, die Aufbereitungsabgänge, welche seit Anbeginn in Form von Bergehalden und Schlammteichen zwischengelagert wurden, einer möglichst vollständigen Aufbereitung zu marktfähigen, innovativen Mineralprodukten zuzuführen.

Ganz im Sinne des "Zero Waste"-Gedankens sollen in Deponien, Halden und Teichen abgelagerte Rückstände historischer Verarbeitungsprozesse genutzt werden. Nicht unerwähnt bleiben darf daher, dass KAMIG seit jeher bemüht war, die anfallenden Nebenprodukte zumindest teilweise zu nutzen. So werden nennenswerte Anteile der quarzreichen Sandfraktion seit Jahren in der Bauindustrie genutzt. Bereits Mitte des vergangenen Jahrhunderts wurde an der Nutzbarmachung der Aufbereitungsabgänge, insbesondere der darin enthaltenen Quarzinhalte, geforscht. Prof. Steiner zeigte in einem Forschungsbericht aus dem Jahr 1968 [3] dazu Möglichkeiten auf, wobei die betriebsintern als "Schlicker" bezeichneten Feinberge der KAMIG mittels einer Kombination aus anionischer- und kationischer Flotation aufbereitet werden können und dabei Glassandkonzentrate mit $\mathrm{Fe}_{2} \mathrm{O}_{3}$-Gehalten $<300$ ppm hergestellt werden können.

Diese Veröffentlichung dient als Einführung in das Dissertationsprojekt des Erstautors und soll die Aufgabenstel- lung und erste Erkenntnisse aus den bisher durchgeführten Untersuchungen präsentieren.

\section{Aufgabenstellung}

Die Schwerpunkte des gegenständlichen Projekts liegen auf der Entwicklung eines geeigneten und innovativen Aufbereitungsverfahrens, welches in der Lage sein muss, die Lagerstätteninhalte primären und sekundären Ursprungs und die darin in stark schwankender Zusammensetzung enthaltenen Mineralphasen zu markttauglichen Produkten zu verarbeiten. Aus heutiger Sicht muss die zu entwickelnde Aufbereitungsanlage eine wesentliche apparative Erweiterung zum Ist-Stand erfahren. Einerseits soll die Aufbereitungsanlage in der Lage sein, die Herstellung qualitativ hochwertiger Kaolinkonzentrate aus den vorhandenen Sekundärlagerstätten mit vergleichsweise niedrigen Kaolingehalten und erhöhten Störstoffgehalten sicherzustellen, andererseits auch die möglichst vollständige Nutzung der enthaltenen Begleitminerale durch weitere Aufbereitung zu Mineralprodukten von abnahmesichernder Qualität ermöglichen. Dies betrifft konkret die Begleitminerale Quarz, Kalifeldspat, Glimmergruppen- und Schwerminerale, insbesondere jene Schwerminerale, die Seltene-ErdenElemente beinhalten.

\subsection{Kaolin}

Die Kaolinitgehalte des Rohgutes aus den Schlickerteichen und Bergehalden liegen bei $15 \pm 6 \%$ und damit deutlich unter dem Kaolinitgehalt der bislang in Verhieb stehenden Primärlagerstätte, welche außergewöhnlich hohe Kaolinitgehalte von bis zu über $50 \%$ aufweist. (Abb. 1).

Grundsätzlich wird Kaolin als Streichpigment und Füllstoff in der Papierindustrie, als weißbrennendes Rohmaterial für die keramische Industrie (keramische Massen

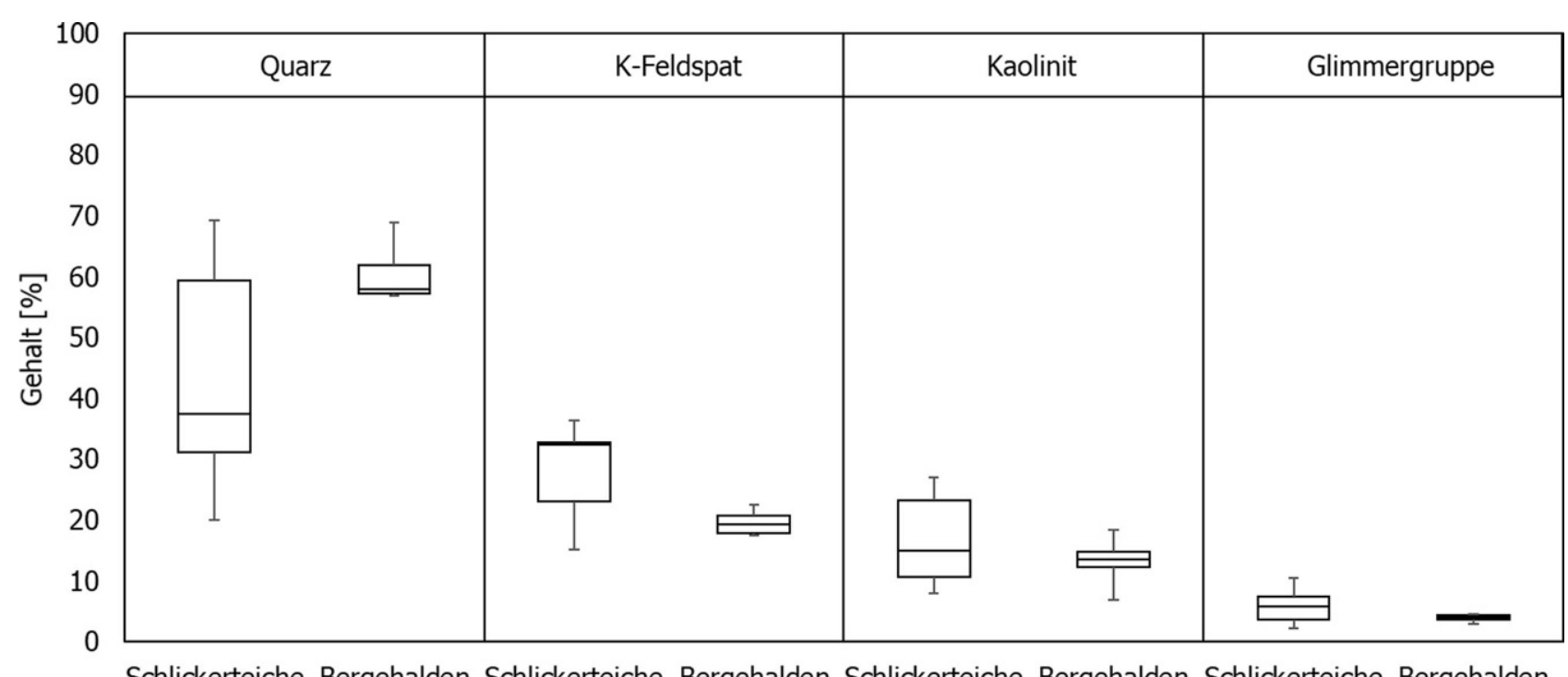

Abb. 1: Gehalte der Hauptmineralphasen der anthropogenen Lagerstättenbereiche am Standort Kriechbaum 


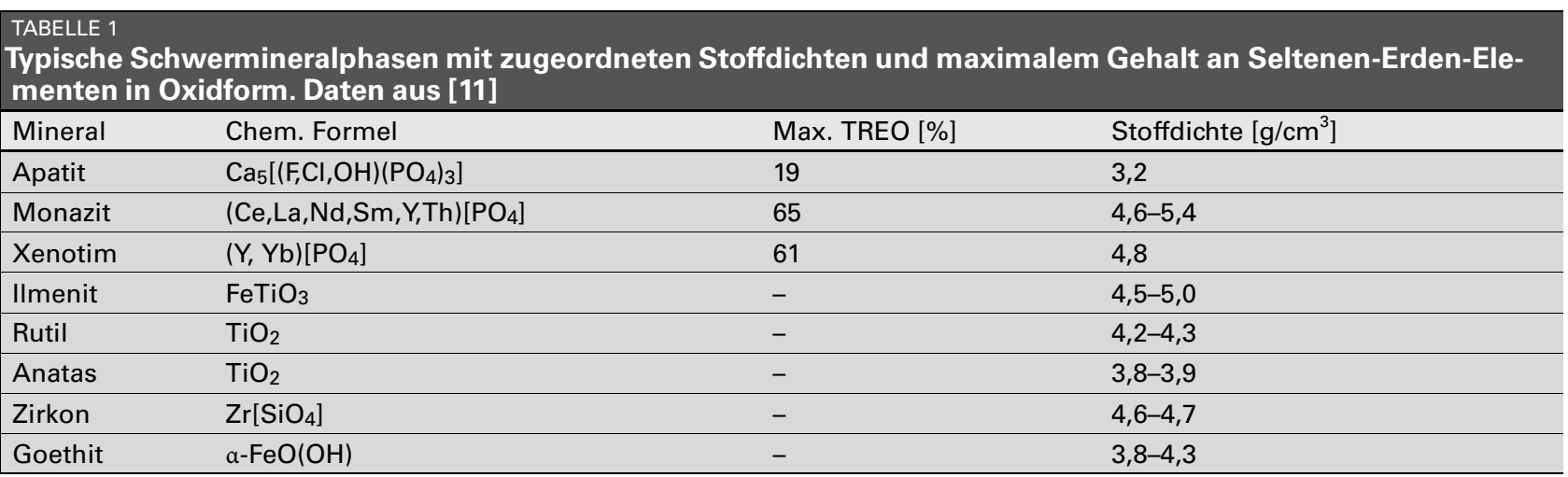

als auch Glasur) eingesetzt. Durch Schwankungen der bereits in der Primärlagerstätte natürlich vorliegenden Mineralphasen unterscheiden sich Rohkaoline stark in ihrer Qualität.

Um geforderte Qualitätskriterien, wie den für oben angeführte Anwendungen essentiellen Weißgrad (meist als R457 oder ISO-Weißgrad angegeben) erfüllen zu können, wurden diverse physikochemische Verfahren zur Weißgradsteigerung von Industriemineralen entwickelt.

Konkret sind den Anforderungen an hohe Weißgrade die Gehalte an Eisen- und Titanoxiden unterschiedlichen Verwitterungsgrades sowie von Organik besonders abträglich. Verfahren zur Abtrennung färbender Eisen- und Titanminerale nutzen in Abhängigkeit von der vorliegenden Mineralphasenzusammensetzung die (Hochgradient-) Magnetscheidung, die Flotation, die selektive Flockung und die reduktive Bleiche oder eine Kombination dieser Prozesse. Aus dem Studium der verfügbaren Literatur geht eine kontinuierliche Intensivierung der Forschungstätigkeiten mit dem Ziel hervor, aus qualitativ minderen Lagerstätten qualitativ höherwertige Kaolinprodukte zu erzeugen [4-6].

Durch die wachstumsfördernde Wirkung von Kaolin sind die vorhandenen Sekundärrohstoffquellen mit pflanzlichem Bewuchs bedeckt und die hieraus erzeugten Kaolinprodukte durch organische Bestandteile verunreinigt, wodurch unzureichende Weißgradwerte resultieren. Um dies zu kompensieren und dennoch marktfähige Weißgrade erzielen zu können, werden Untersuchungen mittels oxidativer Bleiche in Labor- und Pilotmaßstab durchgeführt.

Eine der wesentlichen Fragestellungen umfasst auch, in welcher Dispersität der in den historischen Aufbereitungsabgängen enthaltene Kaolinit vorliegt. Dahingehend ist der aktuell im Einsatz stehende Läuterprozess mittels Schlämmtrommel, Schwerterwäschern und Hydrozyklonklassierung zu evaluieren und an die veränderten Bedingungen anzupassen bzw. allenfalls neu zu gestalten.

Die als Projektziel formulierte Prüfung auf Tauglichkeit ausgewählter Aufbereitungsverfahren und Prozess-Kombinationen bedeutet, bezogen auf die Kaolinfraktion der KAMIG Aufbereitungsabgänge, eine systematisch erarbeitete, vergleichende Gegenüberstellung möglicher Verfahren und Beurteilung unter anderem anhand der erzielten Weißgradsteigerungen, Effizienz der Schadstoffentfrachtung sowie dem Kaolinitausbringen.

\subsection{Quarz}

Der Hauptbestandteil der Sekundärlagerstätten der KAMIG ist Quarz mit stark schwankenden Gehalten von 20-69\%, welcher aktuell weitgehend in niedrigen Preissegmenten seine Anwendung findet (Keramische-, Feuerfest- und Bauindustrie, Sportplatzsand, etc.).

Wesentlicher Teil des Projekts ist, dass die enthaltenen Begleitminerale durch das zu entwerfende Anlagenkonzept künftig in besseren Qualitäten (d.h. deutlich geringeren Schwermineral- oder Kaolingehalten) anfallen sollen und damit höhere Preise erzielen. So soll geprüft werden, ob die Quarzsande auch für höherwertige Anwendungen (z. B. Verpackungs-, Flach- und Spezialglasindustrie) genutzt werden können. In systematischen Untersuchungsreihen unter Einbeziehung etablierter Prozesse wie auch innovativer Apparateentwicklungen soll geprüft werden, inwieweit sich der im Rohgut der KAMIG enthaltene Quarzanteil zu hochwertigen Konzentraten aufbereiten lässt. Konkret bedeutet dies, dass unter anderem mittels Magnetscheidung ausgelotet werden soll, wie weit der $\mathrm{Fe}_{2} \mathrm{O}_{3}$-Gehalt gesenkt werden kann.

\subsection{Kalifeldspat}

Nach Quarz stellt Kalifeldspat den größten Anteil in den Sekundärlagerstätten der KAMIG dar. Im Zuge von mehreren Beprobungskampagnen und folgender Rohgutcharakterisierung konnte der Kalifeldspatgehalt in den Aufbereitungsabgängen der KAMIG mit mittleren Gehalten von 20 bis $28 \%$, je nach Lagerstättenbereich, quantifiziert werden.

Um auch diesen nutzbar zu machen, wird sowohl an verbreiteten Nass-Aufbereitungsprozessen, als auch an trockener Aufbereitung geforscht.

Ein etablierter Prozess und Stand der Technik in der Nassaufbereitung ist die Flotation mit kationenaktiven Sammlern ( $n$-Alkylammoniumsalze) bei $\mathrm{pH} \mathrm{2-3}$ in flusssaurer Trübe nach vorangegangener Attritionsbehandlung und möglichst trennscharfer Entschlämmung. Wenngleich mannigfaltige Herausforderungen in Bezug auf Gesundheit, Sicherheit und Umwelt mit der Verwendung von Flusssäure einhergehen, vermochten sich HF-freie Alternativen bislang industriell nur in Nischenanwendungen durchzusetzen. Die Funktion der Flusssäure in der Feld- 
Abb. 2: REE + Y Potenzial ausgewählter, im Aufbereitungsprozess der KAMIG

anfallenden, Ströme jenen der primären Rohkaolin-

lagerstätte in Kriechbaum

(Top/Mittelteil/Basis) gegen-

übergestellt. Der Unterlauf

der Hydrozyklonklassierung

wird als "Schlicker", das am

Magnetscheider anfallende

Magnetprodukt als "KAMIG

$M P^{\prime \prime}$ bezeichnet. Gehalte laut ICP-MS, [8]

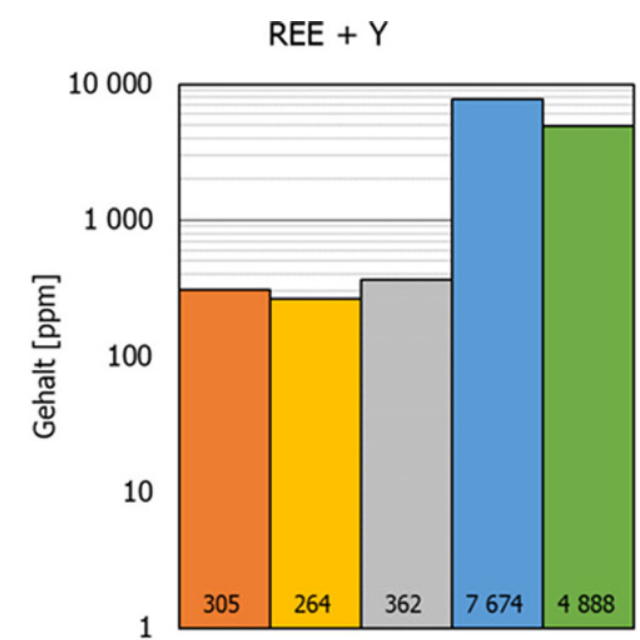

$\mathrm{Ce}$

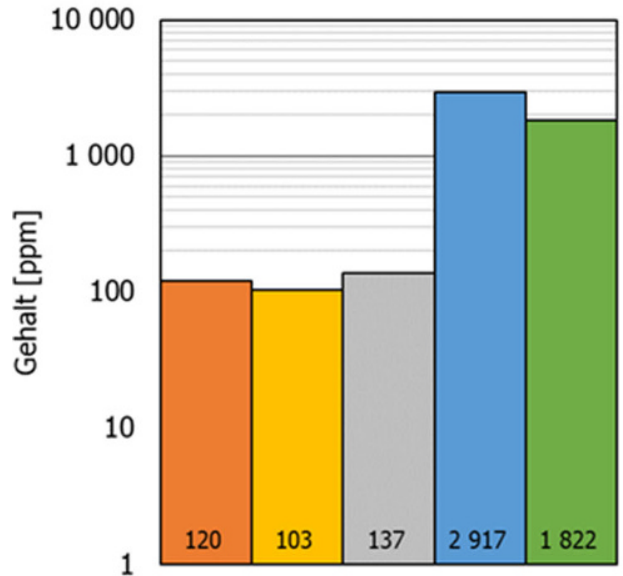

Sm

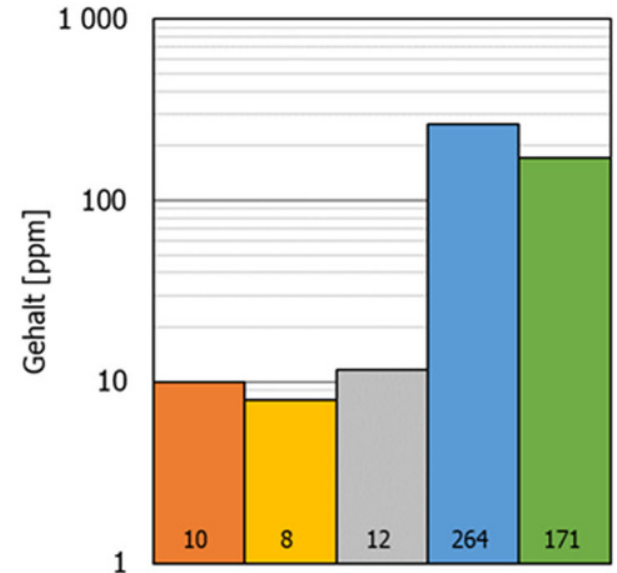

口Rohkaolin - Top $\square$ Rohkaolin - Mittelteil
La

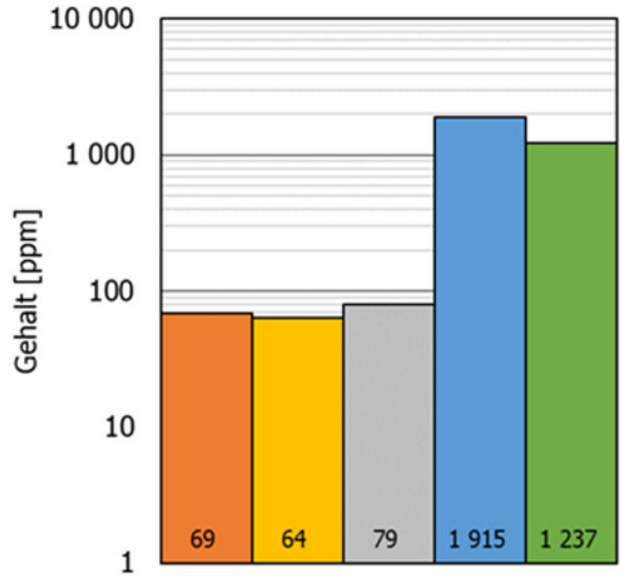

Nd

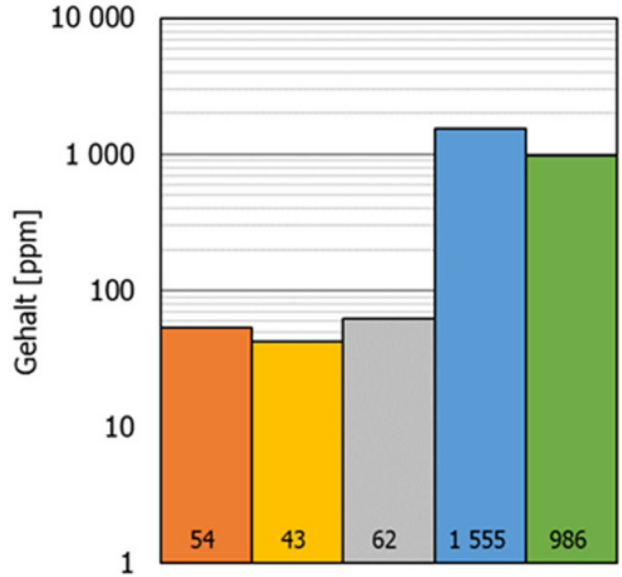

Y

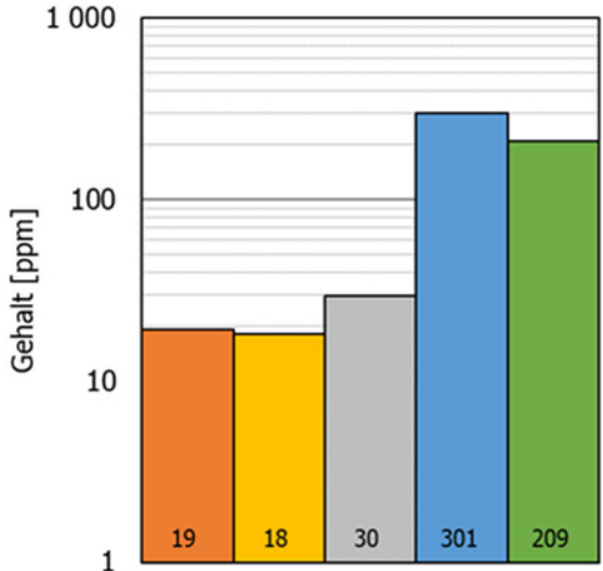

口Rohkaolin - Basis 口Schlicker aKAMIG MP 
Abb. 3: Anreicherung der schweren Seltenen-ErdenElemente (HREE) im Schlicke sowie Magnetprodukt (abgekürzt als KAMIG MP) im aktuellen KAMIG Prozess. Daten aus [8]

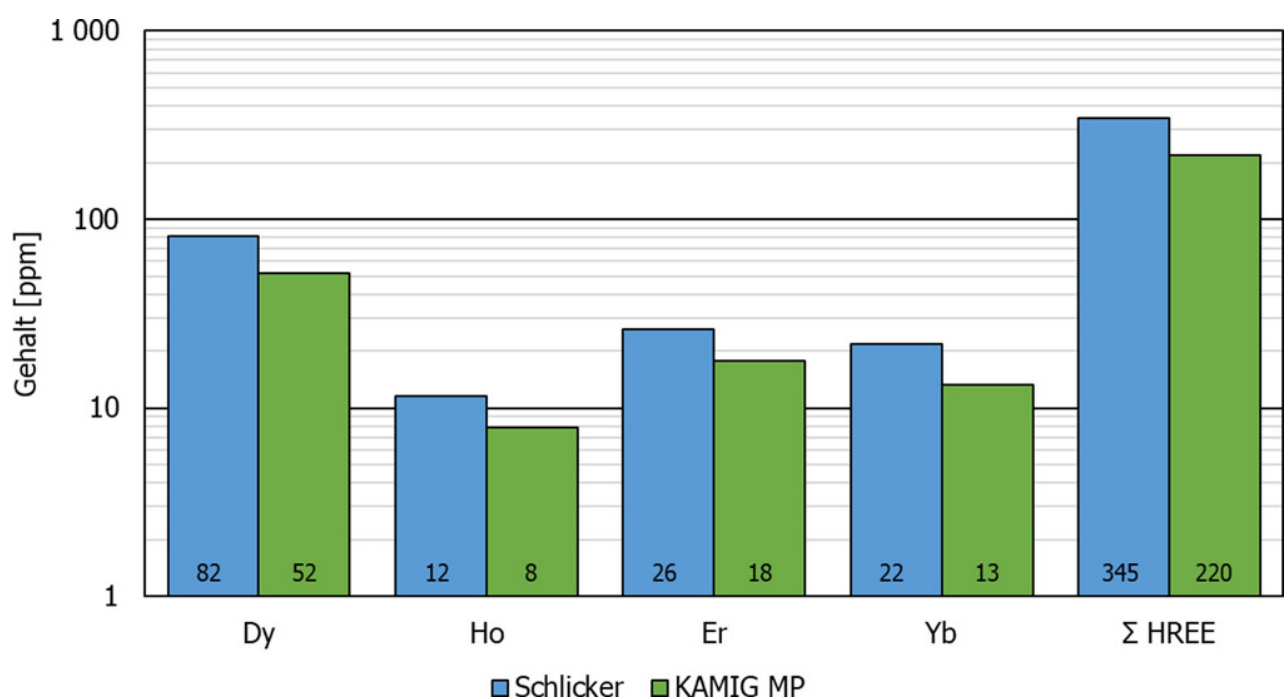

spatflotation ist bereits seit Jahrzehnten Thema zahlreicher Forschungsarbeiten, woraus unterschiedliche potenzielle Wirkungsmechanismen abgeleitet wurden. Im konkreten Forschungsfeld des gegenständlichen Projektes wird als Nassprozess an flusssaurer- als auch HF-freier Flotation geforscht.

Daneben hat sich die trockene Aufbereitung von Industriemineralen mittels Elektroscheidung am Lehrstuhl für Aufbereitung und Veredlung zu einem Forschungsschwerpunkt entwickelt. Das hierzu vorhandene Know-How soll auf die anstehende Trennaufgabe übertragen werden.

Die Ergebnisse der flotativen Quarz-/Feldspat-Trennung werden den Untersuchungsergebnissen der trocken betriebenen Aufbereitungsversuche mittels Elektroscheidung nach triboelektrischer Aufladung einander vergleichend gegenübergestellt.

\subsection{Schwerminerale}

Für Kaolin- bzw. Quarz-/Feldspat-Produkte gelten Schwerminerale üblicherweise als qualitätsmindernde Störstoffe. Jedoch ist abseits der vordergründigen Abtrennung aus diesen Produktströmen im zu konzipierenden Aufbereitungsprozess auch eine Verwertung dieser Schwermineralphasen anzustreben. In Tab. 1 sind typische Schwerminerale und deren maximaler Gehalt an Seltenen-ErdenElementen in Oxidform (total rare earth element oxides; Abk. TREO) dargestellt.

Die in der Primärlagerstätte durchschnittlich vorhandenen $310 \mathrm{ppm}$ an Seltenen-Erden-Elementen (REE + Y) werden weitgehend durch den aktuellen Aufbereitungsprozess über den Unterlauf der Hydrozyklonklassierung (Schlicker) sowie im magnetischen Produkt eines installierten Magnetscheiders (KAMIG MP) aus dem Kaolinprozess ausgeschleust und in den Schlickerteichen und Bergehalden abgelagert. Das Potenzial der enthaltenen Metalle der Seltenen-Erden wurde in mehreren Stoffströmen und Lagerstättenbereichen umfassend erhoben und quantifiziert. Die in Abb. 2 dargestellten Gehalte ausgewählter
Seltenen-Erden-Elemente, sowie in Abb. 3 für ausgewählte schwere Seltenen-Erden-Elemente (heavy rare earth elements, Abk. HREE; Yttrium, Gadolinium - Lutetium) demonstrieren eine bemerkenswerte Anreicherung dieser im Aufbereitungsprozess der KAMIG. Speziell in den angeführten Stoffströmen sind signifikant hohe Gesamtgehalte von $2634-7674$ ppm (entspricht 0,26-0,77\%) an SeltenerdElementen $(R E E+Y)$ analysiert worden [8]. In den angeführten Stoffströmen ergibt sich durch den gegenwärtigen, mit Primärrohstoff betriebenen Kaolin-Aufbereitungsprozess ein Anreicherungsfaktor von 16 (Schlicker) bzw. 25 (Magnetprodukt).

Wie aus Abb. 2 ersichtlich ist, hat der, mit zunehmender Teufe abnehmende Kaolinitisierungsgrad (Rohkaolin: Top Basis der Lagerstätte) kaum Einfluss auf die Konzentration der Seltenen-Erden-Elemente.

Das aktuell im Prozess anfallende Magnetprodukt wird von den Mineralphasen IImenit $79 \%$ in der Korngrößenfraktion $>60 \mu \mathrm{m}$ ) und Monazit ( $>10 \%$ in der Fraktion $>60 \mu \mathrm{m}$ ) dominiert. Speziell im Korngrößenbereich $<60 \mu \mathrm{m}$ liegen Monazitgehalte von über $32 \%$ vor [7].

Schlussendlich gelten für eine wirtschaftliche Nutzung unter alleiniger Betrachtung der Seltenen-Erden-Elemente die im gegenwärtigen, mit Primärrohstoff betriebenen Aufbereitungsprozess anfallenden Massen als zu gering [7]. Aufgrund des sich mittelfristig ändernden Rohgutes und des nahenden Endes der Lebensdauer der bestehenden Aufbereitungsanlage besteht ohnehin die Notwendigkeit der Errichtung einer neuen Aufbereitungsanlage. Hierdurch könnte die Prämisse, dass ein Aufbereitungsprozess exklusiv für enthaltene REE nicht wirtschaftlich darstellbar ist, ungültig werden. So soll neben der technischen Machbarkeit der REE-Aufbereitung auch geprüft werden, inwiefern sich eine Aufbereitung zu Kuppelprodukten unter der geänderten Prämisse wirtschaftlich darstellen lässt.

Konkret bedeutet dies im ersten Schritt, im Rahmen der Merkmalsklassenanalytik spezielles Augenmerk auf diese Schwermineralfraktionen zu legen. Anschließend soll mittels kombinierter Dichtesortierung und Magnetscheidung eine Abtrennung der Seltene-Erden-Elemente beinhalten- 
Abb. 4: Spanne der KorngröBenverteilungen aus den Schlickerteichen Nordwest und Bergehalden am Standort Kriechbaum

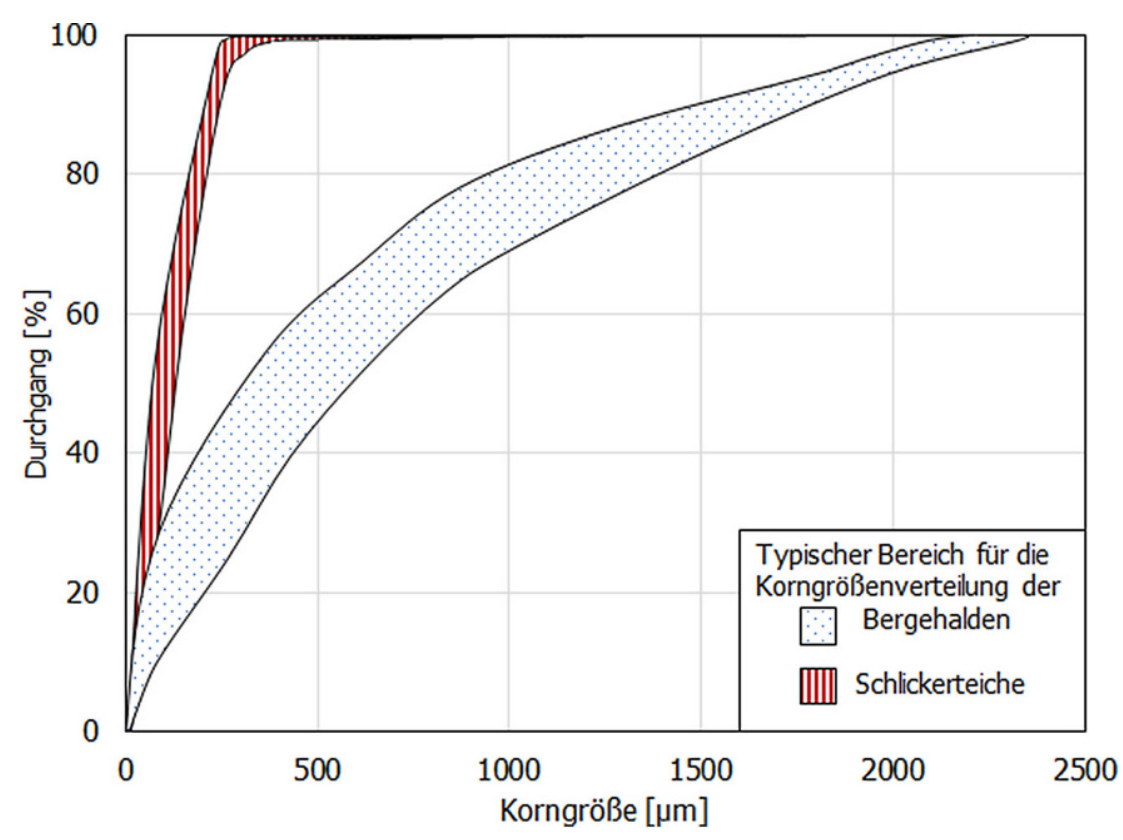

Abb. 5: Vergleichende Gegen überstellung ausgewählter Kennzahlen zur Korngrößenverteilung von den Bergehalden und Schlickerteichen. 20-, $^{2}$ k50- und k80-Werte der Feinfraktion $<63 \mu \mathrm{m}$

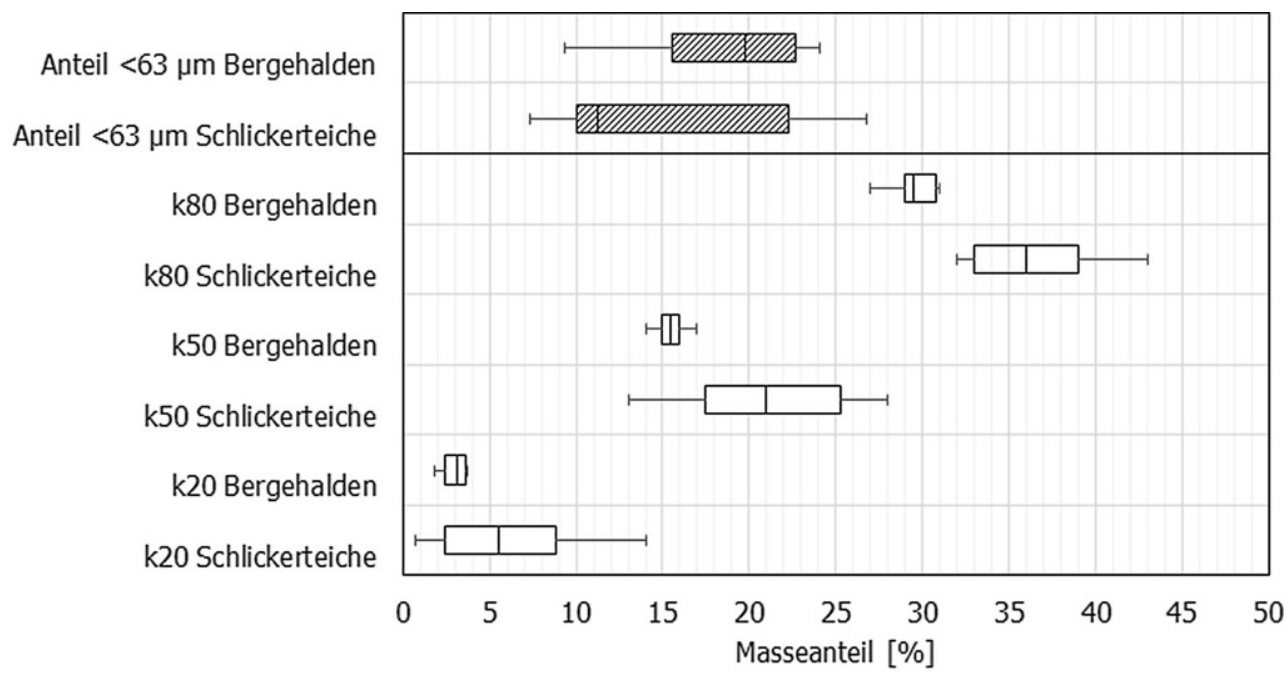

Korngrößenkennwerte der Fraktion <63 $\mu \mathrm{m}[\mu \mathrm{m}]$

Die Schlickerteiche weisen eine wesentlich größere Schwankungsbreite in der Zusammensetzung der Mineralphasen auf und sind generell ärmer an Quarz (43\%) bzw. reicher an Kalifeldspat (28\%) und Kaolinit (17\%) als die Bergehalden, welche sich vornehmlich aus Quarz (60\%), Kalifeldspat (20\%), Kaolinit (13\%) und Glimmer (4\%) als Hauptmineralphasen zusammensetzen [9].

Neben der Mineralogie unterscheiden sich die genannten potenziellen Rohgüter auch signifikant in ihrer Korngrößenverteilung. Siehe Abb. 4, sowie für eine vergleichende Gegenüberstellung der vorgestellten Lagerstättenteile Abb. 5.

- Die untersuchten Einzelproben der Schlickerteiche liegen in einem vergleichsweise engen Kornband vor. Dies gilt insbesondere für den nordwestlichen Teil der Schlickerteiche, wo $100 \%<500 \mu \mathrm{m}$, bei stark schwankenden 
Masseanteilen <63 $\mu \mathrm{m}$ zwischen 22-49\% (im Mittel 32\%) vorliegen.

- Das Rohgut der Bergehalden liegt vergleichsweise gröber, mit etwa $96 \%<2 \mathrm{~mm}$ und einer größeren Spannweite der Einzelproben über sämtliche Korngrößenbereiche hinweg, vor. Hier fallen zwischen 9-24\% (im Mittel $18 \%$ ) in Korngrößenbereichen $<63 \mu \mathrm{m}$ an.

Darüber hinaus zeigten erste Untersuchungen, dass signifikante Kaolinitinhalte auch in groben Dispersitäten vorliegen. In den Schlickerteichen liegen $44 \%$, in den Bergehalden $31 \%$ des Kaolinites in Korngrößenbereichen $>100 \mu \mathrm{m}$ vor [10].

Erste Untersuchungen mittels Automatisierter Mineralogie deuten darauf hin, dass kaum Verwachsungen zwischen Quarz- und Kaolinit vorhanden sind.

\subsection{Aufbereitungsversuche}

Erste Aufbereitungsversuche zur Abtrennung der Schwermineralphasen sowie Untersuchungen zur Quarz-/Feldspat-Trennung zeigten vielversprechende Resultate. In weiterer Folge gilt es diese Untersuchungen über statistisch geplante Versuchsreihen systematisch zu vertiefen. Darauf sollen die Grundlagen für das Konzept eines neuen Aufbereitungsverfahrens für die KAMIG basieren. Die geplante Versuchstätigkeit soll einerseits im Labor- und Technikumsmaßstab am Lehrstuhl für Aufbereitung und Veredlung sowie andererseits im Pilotmaßstab bei der KAMIG und bei Maschinen- und Anlagenbauunternehmen durchgeführt werden.

Konkrete Forschungsgebiete bietet das gegenständliche Projekt in der Magnetscheidung und chemischen Bleiche zur Erhöhung des Weißgrades von Kaolinprodukten aus sekundären Rohstoffquellen und der vergleichenden, trocken und nass durchgeführten Sortierung von Quarz und Feldspat sowie in der Veredlung dieser zu marktfähigen, hochqualitativen Mineralprodukten.

Funding. Open access funding provided by Montanuniversität Leoben.

Open Access Dieser Artikel wird unter der Creative Commons Namensnennung 4.0 International Lizenz veröffentlicht, welche die Nutzung, Vervielfältigung, Bearbeitung, Verbreitung und Wiedergabe in jeglichem Medium und Format erlaubt, sofern Sie den/die ursprünglichen Au- tor(en) und die Quelle ordnungsgemäß nennen, einen Link zur Creative Commons Lizenz beifügen und angeben, ob Änderungen vorgenommen wurden.

Die in diesem Artikel enthaltenen Bilder und sonstiges Drittmaterial unterliegen ebenfalls der genannten Creative Commons Lizenz, sofern sich aus der Abbildungslegende nichts anderes ergibt. Sofern das betreffende Material nicht unter der genannten Creative Commons Lizenz steht und die betreffende Handlung nicht nach gesetzlichen Vorschriften erlaubt ist, ist für die oben aufgeführten Weiterverwendungen des Materials die Einwilligung des jeweiligen Rechteinhabers einzuholen.

Weitere Details zur Lizenz entnehmen Sie bitte der Lizenzinformation auf http://creativecommons.org/licenses/by/4.0/deed.de.

\section{Literatur}

1. Reichl, C.; Schatz, M.; Zsak, G.: World Mining Data 2019, Wien, 2019

2. Kaltenreiner, E.: Aufbereitung in Österreich: Entwicklung und Stand der Kaolinaufbereitung in Österreich, Perg: Eigenverlag, 2011

3. Steiner, H.-J.: Orientierende Untersuchungen über die Aufbereitbarkeit von KAMIG-Sanden. Forschungsbericht von Prof. H.J. Steiner. Institut für Aufbereitung, Montanuniversität Leoben, 1968

4. Forkel, G.; Gassenhuber, R.: Verfahren zur Herstellung aufgehellter Mineralien, 1997

5. Luz, A. B.; Midea A.: Purification of kaolin by selective flocculation: Fifth UBC-McGill Biennial International Symposium, 43rd Annual Conference of Metallurgists of CIM 43 (2004)

6. Mathur, S.: Kaolin Flotation, Journal of Colloid and Interface Science 256 (2002), Nr. 1, S. 153-158

7. Schedl, A.; Benold, C.; Neinavaie, H.; Lipiarski, P.; Hobiger, G.: Potenziale kritischer Rohstoffe III (SEE-Potenziale ausgewählter Kaolin-, Ton-, Leukophyllit- und Grafitvorkommen in Niederösterreich und Oberösterreich: Jahresendbericht Projekt Ü-LG-67_2015), Wien, 2016

8. Höhn, S.; Frimmel, H. E.; Pašava, J.: The rare earth element potential of kaolin deposits in the Bohemian Massif (Czech Republic, Austria), Mineralium Deposita 49 (2014), Nr. 8, S. 967-9

9. Spieß, V.: Prüfung auf Aufbereitbarkeit von Rohkaolinen unter besonderer Berücksichtigung der enthaltenen Begleitminerale, Masterarbeit, Leoben, Montanuniversität Leoben, Lehrstuhl für Aufbereitung und Veredlung, 2019

10. Gasser, W.: Aufbereitungstechnische Untersuchungen an diversen kaolinhältigen Rohgutproben primären und sekundären Ursprungs, Masterarbeit, Leoben, Montanuniversität Leoben, 2019

11. Pohl, W.: Economic geology: Principles and practice; metals, minerals, coal and hydrocarbons; introduction to formation and sustainable exploitation of mineral deposits, 1. ed., Chichester: WileyBlackwell, 2011

Hinweis des Verlags. Der Verlag bleibt in Hinblick auf geografische Zuordnungen und Gebietsbezeichnungen in veröffentlichten Karten und Institutsadressen neutral. 